\title{
Identificación por imágenes del paciente con pie diabético del tipo de lesiones que requirieron o requerirán amputación
}

\author{
Identification by images of the patient with diabetic foot of the type \\ of injuries that required or will require amputation
}

\author{
King-Martínez AC,* Doger-Echegaray P, ${ }^{\ddagger}$ Hoyo-Pérez LI ${ }^{\S}$
}

Hospital General «Dr. Manuel Gea González».

RESUMEN. Introducción: La diabetes es una enfermedad crónico degenerativa que se desarrolla posterior a una disfunción del páncreas. Se estima que actualmente hay 422 millones de personas en el mundo que cuentan con el diagnóstico de diabetes. Una de sus complicaciones más frecuentes es el pie diabético. Se estima que alrededor de 15\% de los pacientes diabéticos tendrán úlceras en las extremidades inferiores. Material y métodos: Es un estudio observacional, descriptivo, prospectivo y transversal. Su objetivo es conocer cuáles son las lesiones iniciales que desencadenaron una amputación en un pie diabético complicado. A los pacientes se les mostraron fotografías clínicas con las imágenes típicas de las patologías para su reconocimiento. Resultados: Se incluyó un total de 50 pacientes entre Enero de 2017 y Julio de 2019. De éstos, 78\% (38) pacientes fueron masculinos, 22\% (11) femeninos. 22 pacientes (44\%) reconocieron el inicio del pie diabético con una úlcera, 13 con flictenas (26\%), 10 con hiperqueratosis $(20 \%)$, cinco con dedos en martillo (10\%). Conclusiones: $96 \%$ de los pacientes lograron identificar al menos una lesión como factor predisponente para la aparición de pie diabético complicado. La presentación de fotografías clínicas resultó ser una herramienta aceptada por los pacientes. Mediante la identificación de su lesión inicial podemos determinar qué lesión
ABSTRACT. Introduction: Diabetes is a chronicdegenerative disease that develops after pancreatic dysfunction. An estimated 422 million people in the world are currently diagnosed with diabetes. One of its most common complications is diabetic foot. It is estimated that about $15 \%$ of diabetic patients will have lower extremities ulcers. Material and methods: It is an observational, descriptive, prospective and cross-sectional study. It aims to know what the initial injuries that triggered an amputation in a complicated diabetic foot. Patients were presented with clinical photographs with images typical of pathologies for recognition. Results: A total of 50 patients were included between January 2017 and July 2019. Of these 78\% (38) patients were male, 22\% (11) female. 22 patients (44\%) recognized as the onset of diabetic foot an ulcer, 13 with blisters $(26 \%), 10$ with hyperkeratosis (20\%), 5 with hammer toes (10\%). Conclusions: $96 \%$ of patients were able to identify at least one injury as a predisposing factor for the onset of their complicated diabetic foot. The presentation of clinical photographs proved to be a patient-accepted tool. By identifying their initial injury we can determine which injury and where, where, it gave rise to the complication that led them to amputation. This information can help to perform

\section{Nivel de evidencia: III}

\footnotetext{
* Jefe de la División de Ortopedia.

‡ Médico pasante del Servicio Social de la División de Ortopedia.

${ }^{\S}$ Médico Residente de la División de Ortopedia.
}

Hospital General «Dr. Manuel Gea González».

Dirección para correspondencia:

Ana Cristina King-Martínez

Jefe de la División de Ortopedia del Hospital General «Dr. Manuel Gea González».

Puente de Piedra Núm. 150, Torre 2, Consultorio 118, Col. Toriello Guerra, C.P. 14050, Alcaldía Tlalpan, CDMX.

Tel: 5556660808 y 5554243165

E-mail: anacristina.king@kingortopedia.com 
y en qué sitio dio origen a la complicación que los llevó a la amputación. Esta información puede contribuir a tomar medidas preventivas para limitar las amputaciones en los miembros pélvicos de los pacientes diabéticos.

Palabras clave: pie diabético, úlceras, complicación, amputación, tratamiento.

\section{Introducción}

La diabetes es una enfermedad crónico degenerativa que se desarrolla posterior a una disfunción del páncreas, ya que éste no produce la insulina suficiente o el organismo no utiliza de manera eficaz la que produce. Se entiende como una patología compleja que requiere un manejo multidisciplinario para disminuir los factores de riesgo.

Se estima que el número de personas con el diagnóstico de diabetes en el mundo ha aumentado de 108 millones en 1980 a 422 millones en 2014. ${ }^{1}$ De acuerdo con la Encuesta Nacional de Salud y Nutrición (ENSANUT) se reportó que la prevalencia de diabetes en México aumentó de $9.2 \%$ en 2012 a $9.4 \%$ en 2016 y $10.3 \%$ en 2018 . Es más prevalente en mujeres con $11.4 \%$ ante $9.1 \%$ en los hombres. ${ }^{2}$ Como podemos ver, a lo largo del tiempo las cifras de personas con este padecimiento han ido en aumento.

Los pacientes sin control glucémico con el tiempo pueden llegar a padecer patologías cardiovasculares, de los vasos sanguíneos, oftálmicas, daño renal así como de los nervios periféricos. Dentro de las complicaciones más frecuentes se encuentran los problemas de la visión $54.5 \%$, daño de la retina en $11.2 \%$, pérdida de la visión con $9.9 \%$ y úlceras en $9.1 \%$ en uno de cada 10 pacientes con diabetes. Las amputaciones se observan en 5.5\% de los pacientes con este padecimiento.

Una de las complicaciones frecuentes es la polineuropatía diabética, comúnmente conocida como neuropatía diabética (NPD), donde hay daño difuso de los nervios periféricos. El pie diabético es una de las complicaciones más frecuentes de la NPD.

El pie diabético se define como la infección, ulceración y destrucción de tejidos profundos asociados con anormalidades neurológicas (pérdida de sensibilidad al dolor) y vasculopatía periférica de diversa gravedad. Se estima que aproximadamente $15 \%$ de los pacientes diabéticos a lo largo de su enfermedad tendrán úlceras en las extremidades inferiores; $7.5 \%$ de estos pacientes que presenten una úlcera, subsecuentemente desarrollarán otra y un tercio de estas úlceras conllevarán a la amputación de la extremidad. ${ }^{3}$

Contamos con varios factores de riesgo que son predictivos para las úlceras y las amputaciones. Dentro de los más importantes tenemos ulceraciones previas, neuropatía, deformidades del pie (hiperqueratosis, dedos en martillo, hallux valgus) y enfermedad vascular. El International Working Group on the Diabetic Foot (IWGDF) desarrolló un sistema para clasificar el pie en riesgo: ${ }^{4}$ preventive measures to limit amputations in the pelvic limbs of diabetic patients.

Keywords: Diabetic foot, ulcers, complications, amputation, treatment.

- Grupo 0: sin evidencia de neuropatía.

- Grupo 1: neuropatía presente, pero sin evidencia de deformidades del pie o enfermedad vascular periférica.

- Grupo 3: neuropatía con evidencia de deformidad del pie o enfermedad vascular periférica.

- Grupo 4: antecedentes de ulceración en el pie o amputación de las extremidades inferiores.

Gran parte de la población de México se encuentra en riesgo de padecer diabetes mellitus tipo 2. Según la encuesta de ENSANUT 2018, 76.8\% de las mujeres mayores de 20 años y 73\% de los hombres presentan obesidad y sobrepeso. ${ }^{2}$ Se han encontrado 19.2 millones de adultos con un índice de masa corporal (IMC) mayor de $25 \mathrm{~kg} / \mathrm{m}^{2}$ que tienen uno o más factores de riesgo. En México cerca de 3.5 millones de personas padecen de diabetes sin diagnosticar. ${ }^{5}$

A lo largo del tiempo la incidencia de la diabetes ha ido en aumento así como el riesgo de padecer alguna de sus complicaciones. A nivel mundial se han ido desarrollando medidas de prevención, detección y tratamiento oportuno que han logrado disminuir los costos de la enfermedad limitando sus complicaciones. Lograr prevenir las complicaciones graves de la enfermedad es una de las metas internacionales con la cual se busca no sólo disminuir el número de amputaciones, sino retrasar el tiempo de aparición de patologías en el pie así como la recurrencia de las mismas.

De acuerdo con la OMS, más de 40\% de los recursos en salud de los países en vías de desarrollo se destinan para la creación de políticas y sistemas de vigilancia, lo que vuelve fundamental tomar medidas para la prevención de esta patología. ${ }^{1}$

Actualmente no se cuenta con un estudio en nuestro país que nos ayude a determinar cuáles son las lesiones que los pacientes pueden presentar en los pies y que con mayor frecuencia se complican hasta llevarlos a una amputación.

El propósito de este estudio es poder determinar las lesiones iniciales que en nuestra población llevaron a los pacientes a desarrollar pie diabético complicado cuya resolución fue una amputación mayor.

\section{Material y métodos}

Se trata de un estudio de diseño observacional descriptivo, prospectivo y transversal. Su objetivo es conocer cuáles son las lesiones iniciales que evolucionan a una amputación en pacientes diabéticos, mediante el reconocimiento de las lesiones por los pacientes a través de imágenes. 


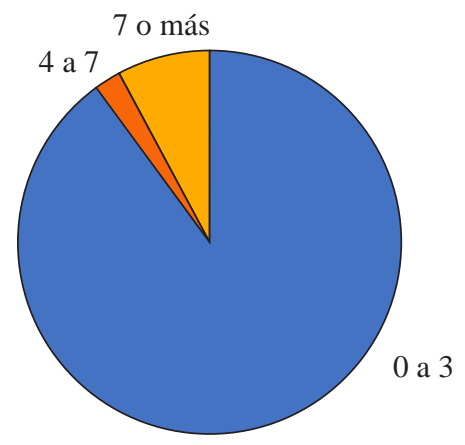

Figura 1: Número de tomas de glicemias capilares por semana.

El estudio se realizó en pacientes de la consulta externa de pie diabético, hospitalización y del área de urgencias, candidatos a amputación mayor (transmetatarsiana, transtibial o supracondílea) por complicaciones de pie diabético de la división de ortopedia del hospital. Se contó con un total de 50 pacientes, los cuales fueron entrevistados entre Enero de 2017 y Julio de 2019.

El hospital cuenta con una amplia población de pacientes diabéticos dentro de los diferentes servicios. Se identificaron sólo a aquéllos que fueron diagnosticados con pie diabético complicado y que ameritaron amputación de miembro pélvico (trasnfemoral, transtibial o transmetatarsiana) como parte de su tratamiento durante el internamiento actual.

A estos pacientes se les invitó a participar, se les realizó una descripción del protocolo, de su objetivo, se les presentó y explicó una carta de consentimiento informado para su estudio.

Se utilizaron fotografías representativas para que los pacientes pudieran identificar las lesiones y deformidades que definen el pie en riesgo. Estas fotografías fueron obtenidas por los investigadores y se imprimieron en calidad fotográfica, se ordenaron en bloques para que éstas fueran mostradas al paciente. A través de las fotografías los pacientes podían identificar la lesión inicial que desencadenó las complicaciones que los llevaron a la amputación. La serie de fotografías a mostrar se ordenó por bloques, de esta manera los pacientes podían ver varias imágenes de diferentes presentaciones de una misma lesión y así lograr una mejor identificación. Dentro de las imágenes a mostrar se incluyeron las lesiones vistas con más frecuencia en el hospital incluyendo las úlceras, hiperqueratosis, dedos en martillo, flictenas y hallux valgus y que la bibliografía refiere como inicio de las complicaciones en el pie diabético.

A todos los pacientes se les mostró el mismo juego de fotografías en el mismo orden. El proceso se explicó de manera clara a los pacientes por medio de los investigadores.

\section{Resultados}

Se reclutó un total de 50 pacientes de forma secuencial. De estos 50 pacientes, 39 fueron de sexo masculino (78\%), mientras que 11 fueron del sexo femenino (22\%). La edad promedio en la que se presentaron las amputaciones fue de
59.46 años, siendo el más joven un paciente de 27 años y el más añoso de 84.

El tiempo de evolución de diabetes mellitus tipo 2 desde el diagnóstico hasta el momento de la amputación varió, siendo el mayor tiempo de evolución 30 años. Tres pacientes mencionaron desconocer el tiempo de evolución de su padecimiento, uno de ellos fue diagnosticado como diabético durante el internamiento en el que se le propuso la amputación. En cuanto al control de la enfermedad los resultados fueron acorde a lo reportado en la literatura, ya que 41 pacientes mencionaron acudir de cero a tres consultas anuales y llevar mal control de su enfermedad. Seis pacientes refirieron acudir a más de nueve consultas anuales y llevar adecuado control de su enfermedad, mientras que tres pacientes mencionaron ir de tres a seis consultas al año. Asimismo, 45 de los 50 pacientes mencionaron tomas de glicemia capilar, máximo tres veces por semana (Figura 1).

En cuanto a la hemoglobina glucosilada contamos con un promedio de $9.34 \%$, siendo la más alta reportada de $15.3 \%$. Sin embargo, no es un dato constante debido a las condiciones de ingreso de los pacientes; de los 50 pacientes 18 no contaron con este estudio durante su internamiento. La causa más frecuente de la falta de procesamiento del estudio fue la baja hemoglobina al momento de la toma de muestra. Dentro de las amputaciones sólo fueron tomados en cuenta los pacientes a los que se les realizó una amputación mayor ya sea transmetatarsiana, transtibial o supracondílea. Los pacientes con amputaciones digitales fueron excluidos del estudio. El número de amputaciones fue de un total de 50, dentro de las cuales 22 fueron transtibiales (44\%), 22 supracondíleas (44\%) y tan sólo seis fueron transmetatarsianas (12\%). De este total de amputaciones 33 (66\%) fueron de miembro pélvico derecho y 17 (34\%) fueron de miembro pélvico izquierdo.

El reconocimiento fotográfico de las lesiones del pie en riesgo se dio en $96 \%$ de los pacientes, dos pacientes no reconocieron ninguna lesión como el inicio del pie diabético. Asimismo, los pacientes pudieron reconocer si esta lesión fue o no el inicio del pie diabético complicado. De los 50 pacientes, 22 (44\%) reconocieron el inicio del pie diabético complicado con una úlcera, 13 con flictenas (26\%), 10 con hiperqueratosis (20\%), cinco con dedos en martillo (10\%)

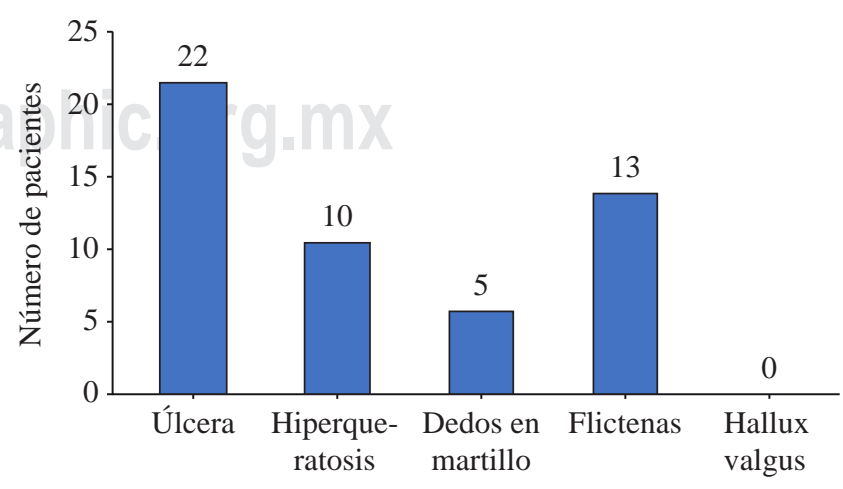

Figura 2: Sitio de inicio del pie diabético. 


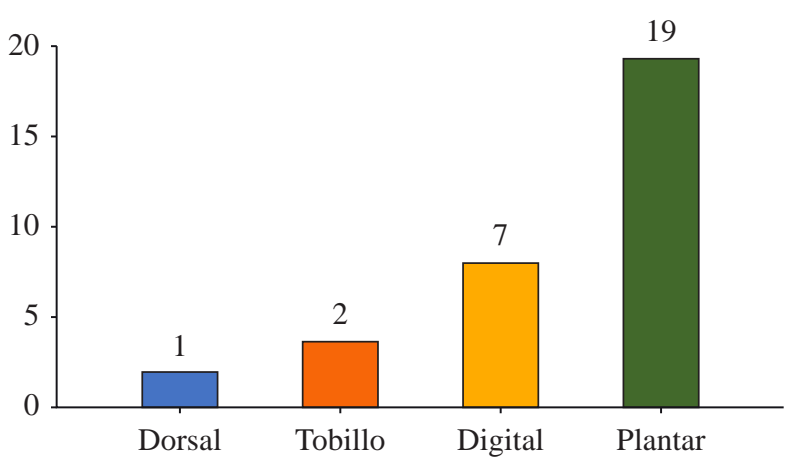

Figura 3: Topografía más frecuente de úlceras.

y ninguno reconoció el hallux valgus como el inicio de su padecimiento (Figura 2). Dentro de las úlceras, que fue la lesión más común encontrada, se observó que la topografía más habitual fue la región plantar del pie, seguida de la región digital, el tobillo y por último la región dorsal (Figura 3). Después de las úlceras, las lesiones que con más frecuencia se identificaron fueron las flictenas. De los 13 pacientes que identificaron a éstas como la lesión de inicio, 53\% las ubicaron en la región dorsal, 18\% en la región digital, $17 \%$ en la región plantar y $12 \%$ en el tobillo.

De los 10 pacientes que identificaron la hiperqueratosis como la lesión de inicio del pie diabético complicado, $61 \%$ la identificaron en la región plantar, mientras que 39\% en la región dorsal. De estas lesiones, 90\% se localizaron en antepié, mientras que sólo $10 \%$ se localizaron en retropié.

En cuanto a los dedos en martillo y el hallux valgus se reportaron números muy bajos identificados como el sitio de inicio del pie diabético complicado. Sólo cinco pacientes identificaron los dedos en martillo como el sitio de inicio, mientras que ningún caso reportó hallux valgus como inicio del pie diabético complicado.

Es de suma importancia el reconocimiento de las lesiones iniciales que conllevan a la complicación de un pie diabético. Tanto el médico como el paciente deben ser capaces de identificarlas con el fin de tratar de lograr prevenirlas. Para ofrecer una mejor calidad de vida a los pacientes diabéticos es necesario reconocer las lesiones que más frecuentemente podemos encontrar en los pies diabéticos complicados. Esto puede dar lugar a medidas para tratar de prevenir el desarrollo de los factores de riesgo modificables así como mejorar la higiene de los pies y recomendar el uso de un calzado apropiado para ellos. Todas estas medidas con el propósito de prevenir estas lesiones.
Al desarrollar este estudio pudimos darnos cuenta de que pese a que la mayoría de los pacientes mencionaron acudir mensualmente a consultas para el control de su padecimiento, tuvieron un mal apego al tratamiento comprobable por las altas cifras de hemoglobina glucosilada y lo avanzado de las complicaciones con las que se presentan en el área de urgencias. El uso de imágenes como herramienta para el diagnóstico, identificación y tratamiento de las lesiones en el pie no se ha considerado como una herramienta para el control del pie diabético. Sin embargo, pudimos darnos cuenta de que $96 \%$ de los pacientes lograron identificar al menos una lesión como factor predisponente de la aparición del pie diabético complicado. Es importante reconocer que es común la aparición de más de una lesión debido a la etiopatogenia del pie diabético.

Es importante que nosotros y nuestros pacientes puedan identificar tempranamente las lesiones que pueden llevar a una lesión mayor en el pie de los diabéticos. Si logramos que tanto personal médico como los pacientes identifiquen estas lesiones y acudan oportunamente a buscar ayuda médica, podemos actuar tempranamente sobre estas lesiones evitando su evolución y complicaciones hasta que la amputación de una extremidad sea el único tratamiento disponible para nuestros pacientes.

\section{Conclusiones}

Llegamos a la conclusión de que el uso de fotografías clínicas para su reconocimiento es una herramienta útil, aceptada por los pacientes y adecuada para que nos proporcione la información necesaria para poder identificar y determinar la incidencia de lesiones, así como su topografía más común y como ésta contribuyó a la amputación de un miembro pélvico.

Bibliografía

1. Diabetes Who.int. (2019). Diabetes. [Online] [Accessed 12 Dec. 2019]. Available at: http://www.who.int/es/news-room/fact-sheets/ detail/diabetes.

2. Who.int. (2019). Diabetes. [Online] [Accessed 12 Dec. 2019] Available at: https://www.who.int/news-room/fact-sheets/detail/ diabetes.

3. Latest diabetes statistics in Mexico-ENSANUT 2018. Anon, (2019). [Online] [Accessed 12 Dec. 2019] Available at: http:// oment.uanl.mx/latest-diabetes-statistics-in-mexico-ensanut2018/?lang=en.

4. Márquez-Godínez SA, Zonana-Nacach A, Anzaldo-Camposa MC, Muñoz-Martínez JA. Riesgo de pie diabético en pacientes con diabetes mellitus tipo 2 en una unidad de medicina de familia. Semergen. 2014. http://dx.doi.org/10.1016/j.semerg.2013.10.004.

5. McCulloch DK. Evaluation of the diabetic foot. UpToDate. 